\title{
ON THE CAUSE OF NUCLEAR JAUNDICE IN NEONATAL SEPSIS WITH JAUNDICE
}

\author{
BY \\ J. I. DE BRUYNE, M.D. and S. VAN CREVELD, M.D. \\ (From the Pediatric Clinic of the Municipal University of Amsterdam)
}

The discovery of the Rhesus factor, which plays such an important part in the explanation of haemolytic disease in the newborn, has also drawn attention to the question of nuclear jaundice and suggested new ideas about its pathogenesis. The possibility has been considered that the erythrocytes which agglutinate as a consequence of the antigenantibody reaction, form agglutination-tbrombi in the capillaries of the brain (Wiener, 1946), but this conception is not generally accepted (Parsons, 1947). It is agreed that nuclear jaundice is produced by bile-staining of previously damaged tissue. However, no explanation can be given of the fact that in this process certain nuclei and cell masses of the brain are almost constantly stained, whereas others remain unstained. One explanation assumes that differences in maturity of the brain nuclei at birth are important, while differences in the blood supply of different parts of the brain have been considered by other observers to be significant in this connexion (Biemond and Van Creveld, 1937).

Authors differ in their estimates of the frequency of the occurrence of nuclear jaundice. Cappell (1947) found it in about 30 per cent. of children who died from icterus gravis, while 12 per cent. of the survivors showed neurological symptoms.

Nuclear jaundice does not occur in congenital acholuric jaundice, nor as a rule in other forms of jaundice of the newborn which are not caused by Rh immunization. However, sometimes nuclear jaundice has been observed after septic jaundice of the newborn. In these cases the first question should be whether $\mathbf{R h}$ immunization can be demonstrated. We have considered this in two recent cases, and have also established the $R h$ status of the parents of two infants who died some years ago of nuclear icterus associated with septic jaundice. An account of these two children was published in 1937 before the discovery of $\mathrm{Rh}$ groups (Biemond and Van Creveld, 1937).

\section{Case Histories}

Case 1 concerns both parents of the child E.C., described in 1937. This child was born in 1933 and was the sixth of healthy parents. The first three children were born at full term and were healthy. Then twins were born, of which one is alive and healthy, the other having died at home at the age of three days: the exact cause of death is unknown, but the infant may have been icteric soon after birth.

According to the parents the sixth child, our patient, became jaundiced soon after birth. When examined in hospital at four days old it had an umbilical infection and severe jaundice, and by the next day there were clinical symptoms of nuclear jaundice. On the fourth day of life the blood did not show any abnormality indicative of icterus gravis neonatorum.

Between 1933 and the end of 1944 the mother did not become pregnant. In August, 1945, she gave birth to a full-term child who on the second day of life was admitted to the hospital, where it died next day. The child became yellow on the second day of life, and jaundice quickly increased. The urine was normal in colour. There was a praecordial systolic murmur. The spleen was palpable, and the liver edge could be felt. The blood contained fifteen nucleated red cells per 1,000 erythrocytes. Autopsy showed nuclear jaundice. It seems probable that this was a case of icterus gravis neonatorum. In this connexion we were greatly interested in the $R h$ tests on the parents, whose luetic reactions were negative. Repeated examination of the serum of the mother showed Rh agglutinins (titre $1: 4$ ) as well as incomplete $R h$ antibodies (titre 1:16). So it is fairly certain that the child who died in 1945 suffered from icterus gravis neonatorum; and notwithstanding the fact that in the child who died in 1933 the diagnosis of septic jaundice had been made on clinical findings, it is very probable that here also $R h$ immunization played a part in the occurrence of the nuclear jaundice. The fact that the morbus haemolyticus becomes manifest in the fourth or fifth pregnancy (which here cannot be established with certainly) need not weigh against this diagnosis (de Bruyne, 1946).

Case 2 concerns the mother of the child M.H. described in 1937. The father, who suffered from bronchial asthma, has since died. The first two children were normal. The third, M.H., was born 
in 1934 spontaneously, at full term, and was not jaundiced at birth but shortly afterwards became icteric. When taken into the hospital on the fourth day of life the baby was deeply jaundiced; the umbilicus was thickened, red, and infiltrated; above the umbilicus there was infiltration of the abdominal wall. The child was somewhat lethargic. The liver was slightly enlarged, the spleen was not palpable. The urine was dark brown and contained bilirubin. Already on admission there was rigidity of both arms. During the following days the clinical picture of nuclear jaundice became more evident. The blood showed no anaemia and no nucleated red cells. The mean diameter of the erythrocytes was somewhat decreased. The luetic reactions in the blood of parents and child were negative.

The mother had two more pregnancies between 1938 and 1940. In none of these children was there any indication for the diagnosis of foetal erythroblastosis. The first child was a girl with Mongolian idiocy, who was not icteric or anaemic in the neonatal period. The second child, according to the mother, had a spina bifida, and died after some days.

The mother was Rh-negative, and no direct agglutinins could be demonstrated, but incomplete $\mathrm{Rh}$ antibodies were detected in the low titre of 1:4. Though in this case the clinical symptoms did not point to nuclear jaundice caused by erythroblastosis foetalis, it is very remarkable that incomplete antibodies were present in the mother's serum after such a lapse of time. On repetition of the test the observation was confirmed.

Case 3. H. is the second child of healthy parents. The first child is 14 years old; after this child the mother had two pregnancies, each ending in a miscarriage after a three months' gestation. The second child showed no symptoms until fortyeight hours after birth, when parents and nurse observed that it had become icteric. The next day it had slight facial twitches, and on the fourth day the condition became worse: the child did not suck, had a temperature of $105^{\circ} \mathrm{F} .\left(40 \cdot 5^{\circ} \mathrm{C}\right.$.), and showed opisthotonus. When it came into the hospital at the age of four and a half days, it was intensely icteric, and showed the clinical picture of nuclear jaundice: opisthotonus, 'mumbling' of the lips, marked hypertonia of the extremities with claspknife phenomenon, and slow mowing movements of the arms. The liver extended two fingerbreadths below the costal margin; the spleen was slightly enlarged. The umbilical cord had already fallen off and the umbilicus showed slight redness and serous secretion. Examination of the blood showed haemoglobin 98 per cent.; erythrocytes 3,660,000 per c.mm.; leucocytes 15,600 per c.mm. (5 per cent. rod-shaped leucocytes, 54 per cent. polymorphonuclear leucocytes, 35 per cent. lymphocytes; 6 per cent. monocytes; marked toxic granulation). No nucleated red blood corpuscles were seen. Blood culture was negative. The next day the clinical symptoms of nuclear jaundice were still very marked. The umbilicus now showed signs of infection; the wound was treated with penicillin. While the clinical diagnosis seemed to be septic icterus with nuclear jaundice, the examination of the blood groups showed that $R h$ immunization of the mother had taken place. The father's blood group was B CDe, the mother's A CDe, and the child's A CDe. On the fifth day after birth the direct Coombs-test with the child's erythrocytes was positive. In the mother's serum no $\mathrm{Rh}$-agglutinins were found but incomplete anti-D antibodies were present to a titre of $1: 64$.

Case 4. C. van V. was the third child of healthy parents. Two elder children were healthy and had no neonatal jaundice. This child became slightly icteric on the second day of life without any other symptom of disease. The jaundice gradually increased. On the fifth day the general aspect of the child became worse: he did not suck; he vomited, was lethargic and suffered from attacks of sudden crying during which arms and legs were spastically extended. No real convulsions were observed.

When the child was admitted to the Children's Clinic of the Binnengasthuis the skin had an intense orange-yellow colour. The baby cried a good deal. The abdomen was slightly swollen. The umbilical cord had already fallen off. The slightly protruding wound of the umbilicus was inflamed and showed on one side a slight infiltration of the skin. The liver and spleen were not palpable.

Examination of the blood showed: haemoglobin 142 per cent.; erythrocyte count $5,380,000$ per 100 c.mm. of blood; reticulocyte count 23 per 1,000 ; leucocyte count 15,500 per c.mm. (metamyelocytes 6 per cent., rod shaped leucocytes 11 per cent., polymorphonuclear leucocytes 52 per cent.; lymphocytes 28 per cent.; normoblasts 2 per 100 leucocytes). The blood groups appeared to be: father $\mathbf{A}$ Rh-positive, mother $O$ Rh-positive, child A Rh-positive. Later on all three appeared to belong to the subgroup CDe. A blood culture of the child remained sterile.

The patient was treated with 60,000 units of penicillin a day, given in 6 intramuscular injections, and by mouth with $1 \mathrm{~g}$. sulfadiazine daily in eight divided doses, while the umbilicus was dressed with penicillin. During the stay in the hospital the tonic extension-cramps of short duration continued, while in the intervals hypertonia of the muscles was present.

Lumbar puncture showed yellow clear fluid under increased pressure. The Nonne reaction was slightly positive, that of Pandy strongly positive. There was one polymorphonuclear neutrophilic leucocyte per c. $\mathrm{mm}$. and 8 lymphocytes.

Next day the general condition remained about the same; the infected umbilicus quickly improved. On the eighth day of life the temperature, which had been normal, suddenly rose to $105 \cdot 6^{\circ} \mathrm{F}$. $\left(40 \cdot 8^{\circ} \mathrm{C}\right.$.). 
The child did not suck, became cyanotic, and died.

The autopsy was performed by Dr. Witsenburg, of Dr. R. van Dam's pathological department.

LVER. The capillaries were greatly dilated, with much bile pigment between the hepatic cells. The portal spaces contained many leucocytes.

No myeloid tissue was seen.

SPLEEN. The spleen contained much blood; there were small follicles without reactive centres.

Uminucus. The umbilicus was rich in vessels, around which were accumulations of leucocytes. On the surface there were necrotic foci without signs of reaction. The cord was separated.

BRAN. There was clear, icteric staining of the corpus striatum, hippocampus, and dentate nucleus.

Discussion. In this case the nuclear jaundice, based on clinical, haematological, serological, and pathological findings could not be explained by Rhesus immunization.

However, in view of the blood groups of the parents, immunization by group A cannot totally be excluded. The titre of the agglutinins in the serum of the mother was established three months after the death of the child: this proved to be $1: 512$, a rather high value but not definite proof of immunization. The titre of antibodies in the maternal serum can, however, decrease quickly after parturition. The possibility therefore exists that directly after the birth of the child this titre was much higher. If we discard the possibility of an A immunization, we have to accept that in this case the disease was caused by septic infection originating from the umbilicus.

\section{Comment}

In a former publication (Biemond and Van Creveld, 1937), the cases of two infants with nuclear and umbilical infection were presented. The argument that in the families of both children no cases of erythroblastosis foetalis had occurred has now lost its value in one case (case 1). It remains remarkable that in both cases erythroblastaemia and increase of the mean diameter of the erythrocytes were absent. The opinion expressed at that time that these cases proved that nuclear jaundice occurs in septic jaundice of the newborn as well as in erythroblastosis appears now to be less securely founded. Indeed, in three of the four cases communicated here we obtained arguments telling more or less in favour of $\mathbf{R h}$ immunization; in the fourth case no indications for this conception could be found. Though we have to accept in three of the four cases the existence of $R h$ immunization, we cannot neglect the infection which was present in all of them. It is difficult to tell how often infection is present in cases complicated by nuclear jaundice, but in future signs of infection should be looked for in every case. Does infection perhaps promote nuclear jaundice in the presence of icterus gravis ? It is remarkable how frequently, especially in the cases accompanied by nuclear jaundice, the icterus gravis itself takes a less characteristic course, as for instance a retarded beginning of the icterus, absence of erythroblastaemia, etc.

\section{Summary}

In two cases of septic icterus in the newborn combined with nuclear jaundice previously described, the examination of the parents and the clinical anamnesis of the children born since 1937 showed that $R h$ immunization was fairly certain in the first case; while in the second, where no clinical indications for this conception were present, the blood examination also spoke to a certain degree in favour of a $\mathbf{R h}$ immunization. In a third case recently observed the $R h$ relations in parents and patient also pointed to a $\mathbf{R h}$ immunization. In a fourth case, $\mathrm{Rh}$ tests of the parents and patient did not point to $R h$ immunization, but the possibility of immunization by blood group $A$ could not be excluded.

We want to thank Dr. van Loghem in Amsterdam and Dr. Weyers in the Hague most cordially for their kind co-operation in determining the $R h$ tests in these cases.

\section{REFERENCES}

Biemond, A. and Van Creveld, S. (1937). Arch. Dis. Childh., $12,173$.

Cappell, D. F. (1947). Lancet, $2,444$.

de Bruyne, J. I. (1946). Thesis, Amsterdam.

Parsons, L. G. (1947). Lancet, 1, 815.

Wiener, A. S. (1946). Amer. J. clin. Path., 15, 106. 